\title{
Effects of continuous vs interval exercise training on oxygen uptake efficiency slope in patients with coronary artery disease
}

\author{
D.M.L. Prado ${ }^{1}$, E.A. Rocco ${ }^{1}$, A.G. Silva ${ }^{1,2}$, D.F. Rocco ${ }^{2}$, M.T. Pacheco ${ }^{2}$, P.F. Silva ${ }^{1}$ and V. Furlan ${ }^{1}$ \\ ${ }^{1}$ Grupo TotalCare, Amil, São Paulo, SP, Brasil \\ ${ }^{2}$ Universidade Santa Cecília, Santos, SP, Brasil
}

\begin{abstract}
The oxygen uptake efficiency slope (OUES) is a submaximal index incorporating cardiovascular, peripheral, and pulmonary factors that determine the ventilatory response to exercise. The purpose of this study was to evaluate the effects of continuous exercise training and interval exercise training on the OUES in patients with coronary artery disease. Thirty-five patients (59.3 \pm 1.8 years old; 28 men, 7 women) with coronary artery disease were randomly divided into two groups: continuous exercise training ( $n=18)$ and interval exercise training $(n=17)$. All patients performed graded exercise tests with respiratory gas analysis before and 3 months after the exercise-training program to determine ventilatory anaerobic threshold (VAT), respiratory compensation point, and peak oxygen consumption (peak $\mathrm{VO}_{2}$ ). The OUES was assessed based on data from the second minute of exercise until exhaustion by calculating the slope of the linear relation between oxygen uptake and the logarithm of total ventilation. After the interventions, both groups showed increased aerobic fitness $(P<0.05)$. In addition, both the continuous exercise and interval exercise training groups demonstrated an increase in OUES $(P<0.05)$. Significant associations were observed in both groups: 1$)$ continuous exercise training (OUES and peak $\mathrm{VO}_{2} r=0.57$; OUES and $\mathrm{VO}_{2}$ VAT $r=0.57$ ); 2) interval exercise training (OUES and peak $\mathrm{VO}_{2} r=0.80$; OUES and $\mathrm{VO}_{2}$ VAT $\mathrm{r}=0.67$ ). Continuous and interval exercise training resulted in a similar increase in OUES among patients with coronary artery disease. These findings suggest that improvements in OUES among CAD patients after aerobic exercise training may be dependent on peripheral and central mechanisms.
\end{abstract}

Key words: Coronary artery disease; Exercise; Cardiorespiratory fitness; Ventilatory efficiency

\section{Introduction}

Cardiopulmonary exercise testing (CPX) is a highly reliable and well-validated approach to assessing aerobic performance and monitoring exercise tolerance in patients with cardiovascular disease (1). In this context, Baba et al. (2) introduced the oxygen uptake efficiency slope (OUES), an objective and reproducible measure of cardiopulmonary function. The OUES is derived from the single regression analysis between oxygen uptake and minute ventilation during incremental exercise. Importantly, OUES evaluates the functional capacities of several organ systems, such as cardiovascular, pulmonary and skeletal muscle metabolism during exercise $(2,3)$ in a single index. The OUES has been investigated as an index of cardiopulmonary functional reserve in patients with various conditions (4-6). Of particular note, a study that followed patients with cardiovascular disease over 6 years showed that the OUES was a good prognostic indicator (7).
Aerobic exercise training has been recommended as a non-pharmacological treatment for patients with coronary artery disease (CAD) $(8,9)$. In this regard, continuous exercise training (CET) has been shown to promote increased cardiorespiratory fitness in CAD patients (10). However, over the last few decades, there has been increasing interest in interval exercise training (IET) for cardiac rehabilitation $(10,11)$. IET consists of periods of high-intensity exercise alternated with periods at lower intensity; this allows cardiac patients to complete short exercise bouts at a higher intensity than would have been possible during continuous exercise. Previous investigations $(11,12)$ have also shown that IET effectively improves the aerobic fitness of CAD patients.

Although both CET and IET have been shown to improve aerobic fitness, there is little information about the effects of the mode and intensity of exercise training on OUES in CAD

Correspondence: D.M.L. Prado: <danilomprado@.usp.br> 
patients. Thus, the purpose of this study was to evaluate the effects of CET and IET on OUES in patients with CAD.

\section{Subjects and Methods}

\section{Population}

The participants were patients admitted to the coronary care unit of the TotalCor Hospital for the diagnosis of CAD. After being discharged from the hospital, the patients enrolled in a cardiac rehabilitation program at the cardiorespiratory rehabilitation center of the Amil group. Thirty-five CAD patients $(59.3 \pm 1.8$ years old; 28 men, 7 women) were randomly divided into two groups: CET $(n=17)$ and IET $(n=18)$. This trial was designed to test the exercise-training modalities for noninferiority, with the key secondary objective of testing for superiority with respect to the OUES. The inclusion criterion was having stable CAD diagnosed by coronary angiography. Exclusion criteria included having unstable angina pectoris, complex ventricular arrhythmias, pulmonary congestion, and orthopedic or neurological limitations to exercise. Patients remained on their standard medications throughout the study, and no changes in medications were reported. The study was approved by the Ethics Committee of the Universidade Santa Cecilia (66/2011) and all of the study participants gave their written informed consent.

\section{Graded exercise test}

Maximal graded exercise tests were carried out on a programmable treadmill (DigiStress model pulsar, Brazil) before, and three months after commencing the IET or CET intervention. Gas exchange and ventilatory variables were measured continuously during the gas exchange tests, breath-by-breath, using an open-circuit spirometry procedure on an exercise-based system (SensorMedics, model Vmax 229 Pulmonary Function/Cardiopulmonary Exercise Testing Instrument, USA). The following variables were obtained breath-by-breath and expressed as 30-s averages: pulmonary oxygen uptake $\left(\mathrm{VO}_{2} \mathrm{~mL} \cdot \mathrm{kg}^{-1} \cdot \mathrm{min}^{-1}\right.$, standard temperature and pressure, dry) pulmonary ventilation (VE L/min body temperature and pressure, saturated), end-tidal carbon dioxide pressure $\left(\mathrm{PetCO}_{2}\right.$ $\mathrm{mmHg}$ ), ventilatory equivalent ratio for oxygen $\left(\mathrm{VE} / \mathrm{VO}_{2}\right)$, and ventilatory equivalent ratio for carbon dioxide (VE/ $\mathrm{VCO}_{2}$ ). Before each test, the gas analyzers were calibrated using a gas mixture containing known concentrations of carbon dioxide and oxygen balanced with nitrogen, and the flow meter was calibrated using a 3-L syringe. Heart rate was continuously recorded at rest, during the graded exercise testing, and during the recovery period using a 12-lead ECG (HW Systems, HeartWare Ltda, USA). All tests in this study were performed in the same laboratory at a room temperature of $20-23^{\circ} \mathrm{C}$.

The subjects performed a ramp-like progressive exercise test to exhaustion on the treadmill. The exercise workload (speed and/or slope) was increased every
1 minute with completion of the incremental part of the exercise test occurring between 8 and $12 \mathrm{~min}$. The following criteria were used to define maximal effort: 1) participants demonstrated subjective evidence of exhaustion (unsteady gait, facial flushing, and hyperpnea), and either 2) peak heart rate $(H R) \geqslant 95 \%$ age-predicted maximum, or 3 ) maximal respiratory exchange ratio $(R E R) \geqslant 1.10$ (1).

\section{Ventilatory anaerobic threshold}

The ventilatory anaerobic threshold (VAT) was defined as the break point between the increase in the carbon dioxide output and $\mathrm{VO}_{2}$ (V-Slope) or the point at which VE/ $\mathrm{VO}_{2}$ reached its minimum value and began to rise without a concomitant rise in $\mathrm{VE} / \mathrm{VCO}_{2}(13)$.

\section{Respiratory compensation point}

The respiratory compensation point $(\mathrm{RCP})$ was defined as the point at which $\mathrm{VE} / \mathrm{NCO}_{2}$ reached its minimum value and began to rise, and the highest value of $\mathrm{PetCO}_{2}$ before its progressive fall (14). In the present investigation, all patients reached the $\mathrm{RCP}$.

\section{Peak oxygen consumption}

The peak oxygen consumption was defined as the maximum $\mathrm{VO}_{2}$ attained during the exercise period in which the subject reached exhaustion (analog scale from Borg's scale of perceived exertion).

\section{Oxygen uptake efficiency slope calculation}

The OUES was assessed based on the respiratory data during exercise by calculating the slope of the linear relationship between $\mathrm{VO}_{2}$ (y-axis) and the logarithm of $\mathrm{VE}$ (x-axis) using single regression analysis. The OUES was derived from the relationship: $\mathrm{VO}_{2}=a \log 10 \mathrm{VE}+\mathrm{b}$, where $a$ is the OUES and $b$ is the intercept. Before inclusion in the regression analysis, respiratory data were averaged every $30 \mathrm{~s}$ from the beginning of the second minute of exercise until evident exhaustion. The first minute of exercise was not included in the analyses because of irregular breathing patterns during early exercise (2).

\section{Ventilatory efficiency analysis}

The $\mathrm{VE} / \mathrm{VCO}_{2}$ Slope, which reflects the rate of increase in minute ventilation per unit increase in $\mathrm{CO}_{2}$ production, was obtained by linear regression analysis of the relationship between $\mathrm{VE}$ and $\mathrm{VCO}_{2}$ during exercise using the data from the entire exercise test excluding the first minute (15).

The difference in end-tidal carbon dioxide pressure from at rest to its highest value during exercise $\left(\Delta \mathrm{PeTCO}_{2}\right.$ rest-exercise) was also analyzed (1).

\section{Exercise-training program}

A supervised exercise-training program was conducted at the cardiorespiratory rehabilitation center of the Amil Group. The exercise-training program consisted of three 60-min exercise sessions per week for a 3-month 
period. Each exercise session included a 5-min warm-up, 42 or $50 \mathrm{~min}$ of aerobic exercise, and $5 \mathrm{~min}$ of cool-down exercises. The CET consisted of $50-\mathrm{min}$ of treadmill exercise at VAT intensity. The IET consisted of seven sets of $3 \mathrm{~min}$ at RCP and seven sets of $3 \mathrm{~min}$ of exercise at VAT intensity, totaling $42 \mathrm{~min}$ of exercise. The estimated energy expenditure for both exercise-training modalities ranged from 200 to $230 \mathrm{kcal} / \mathrm{min}$. Heart rate was monitored throughout the sessions to ensure that all patients exercised within the intensity limits. Adherence to the exercise-training program was assessed based on the percentage of exercise sessions attended, with all patients attending more than $80 \%$ of the sessions.

\section{Statistical analysis}

The statistical analyses were carried out using SPSS version 16.0 (SPSS Inc., USA). Data are reported as means $\pm S E$. A $P$ value of $<0.05$ was considered to be statistically significant. The normality of distribution was checked for all variables using the Kolmogorov-Smirnov test. Student's unpaired $t$-tests and Fischer's exact tests were used to assess baseline differences between the CET and IET groups for all dependent variables.

Two-way analysis of variance (ANOVA) with repeated measures was performed to test for possible within-group and between-group differences in physical characteristics, cardiorespiratory parameters, and the OUES. When significant differences were detected, Tukey's post hoc comparisons were performed. Single regression analysis was used to investigate the relationship between OUES and cardiorespiratory parameters after exercise training.

For the CET group, the sample size of 18 provided the power $(96 \%)$ to detect a difference in OUES based on a relevant difference of $0.32 \mathrm{~L} / \mathrm{min}$, an $\mathrm{SD}$ of $0.35 \mathrm{~L} / \mathrm{min}$, $\alpha=0.05$, and a two-tailed test of significance. For the IET group, the sample size of 17 provided the power (99\%) to detect a difference in OUES based on a relevant difference of $0.32 \mathrm{~L} / \mathrm{min}$, an SD of $0.30 \mathrm{~L} / \mathrm{min}, \alpha=0.05$, and a two-tailed test of significance.

\section{Results}

\section{Effects of interventions}

Before the interventions, there were no between-group differences in physical characteristics (Table 1). After the interventions, neither the CET nor IET group showed any reduction in body weight (Table 2 ).

With respect to clinical parameters, the CET group had more patients with hypertension than the IET group; however, no between-group differences in left ventricular ejection fraction were observed (Table 1). More patients in the CET group were being treated with beta-blockers than in the IET group.

Both groups had similar levels of aerobic fitness (Table 2). In addition, no statistically significant differences in pre-intervention OUES were present (Table 2).

\section{Cardiorespiratory response}

After the interventions, CAD patients subjected to either CET or IET showed no differences in peak RER or HR. However, both the CET and IET groups showed an increase in $\mathrm{VO}_{2}$ at VAT and peak $\mathrm{VO}_{2}$. Between-group comparisons revealed similar increases in aerobic fitness after the exercise programs (Table 2). The OUES values were similarly and significantly increased from baseline in both the CET and IET groups after the exercise-training program (Table 2).

Table 1. Patient characteristics.

\begin{tabular}{lccc}
\hline & All patients & CET & IET \\
\hline $\mathrm{N}$ & 35 & 18 & 17 \\
Age (years) & $59.3 \pm 1.7$ & $61.3 \pm 2.2$ & $56.5 \pm 2.7$ \\
Weight $(\mathrm{kg})$ & $80.0 \pm 2.3$ & $78.5 \pm 3.2$ & $81.6 \pm 3.7$ \\
$\mathrm{BMI}\left(\mathrm{kg} / \mathrm{m}^{2}\right)$ & $28.3 \pm 0.6$ & $28.0 \pm 1.0$ & $28.5 \pm 0.7$ \\
Men/women & $28 / 7$ & $14 / 4$ & $14 / 3$ \\
Hypertension & $23(66 \%)$ & $15(83 \%)$ & $8(47 \%)^{*}$ \\
Diabetes & $10(29 \%)$ & $4(36 \%)$ & $6(64 \%)$ \\
Myocardial infarction & $15(43 \%)$ & $9(26 \%)$ & $6(17 \%)$ \\
LVEF (\%) & $58.7 \pm 1.4$ & $55.1 \pm 4.1$ & $57.2 \pm 2.0$ \\
CABG & $26(74 \%)$ & $13(72 \%)$ & $13(76 \%)$ \\
PTCA & $9(26 \%)$ & $5(28 \%)$ & $4(24 \%)$ \\
Beta-blockers & $28(80 \%)$ & $17(94 \%)$ & $11(65 \%)^{*}$ \\
ACE- inhibitors & $15(43 \%)$ & $8(44 \%)$ & $7(41 \%)$ \\
\hline
\end{tabular}

Data are reported as means \pm SE or $n(\%)$. CET: continuous exercise training; IET: interval exercise training; BMI: body mass index; LVEF: left ventricular ejection fraction; $C A B G$ : coronary artery bypass grafting; PCTA: percutaneous transluminal coronary angioplasty; ACE: angiotensin-converting enzyme. ${ }^{*} \mathrm{P}<0.05$, CET compared to IET (Fischer's exact test). 
Table 2. Physical and cardiorespiratory parameters in patients with coronary artery disease who performed continuous exercise training or interval exercise training.

\begin{tabular}{|c|c|c|c|c|}
\hline & \multicolumn{2}{|c|}{ CET } & \multicolumn{2}{|c|}{ IET } \\
\hline & Pre & Post & Pre & Post \\
\hline \multicolumn{5}{|l|}{ Physical measurements } \\
\hline Weight $(\mathrm{kg})$ & $78.5 \pm 3.2$ & $78.3 \pm 3.3$ & $81.6 \pm 3.3$ & $81.9 \pm 3.0$ \\
\hline BMI $\left(\mathrm{kg} / \mathrm{m}^{2}\right)$ & $28.0 \pm 1.0$ & $28.0 \pm 1.1$ & $28.5 \pm 0.7$ & $28.5 \pm 0.7$ \\
\hline \multicolumn{5}{|c|}{ Cardiorespiratory measurements } \\
\hline Peak $\mathrm{VO}_{2}\left(\mathrm{~mL} \cdot \mathrm{kg}^{-1} \cdot \mathrm{min}^{-1}\right)$ & $18.8 \pm 1.2$ & $23.0 \pm 1.3^{*}$ & $17.9 \pm 1.0$ & $22.3 \pm 1.1^{*}$ \\
\hline Peak RER & $1.14 \pm 0.2$ & $1.12 \pm 0.2$ & $1.16 \pm 0.2$ & $1.12 \pm 0.2$ \\
\hline Peak HR (bpm) & $124 \pm 4.9$ & $122 \pm 4.1$ & $129 \pm 6.4$ & $131 \pm 4.7$ \\
\hline $\mathrm{VO}_{2}(\mathrm{VAT})\left(\mathrm{mL} \cdot \mathrm{kg}^{-1} \cdot \mathrm{min}^{-1}\right)$ & $12.7 \pm 0.7$ & $15.3 \pm 0.7^{*}$ & $12.3 \pm 0.6$ & $14.0 \pm 0.7^{*}$ \\
\hline OUES (L/min) & $1.82 \pm 0.1$ & $2.17 \pm 0.1^{*}$ & $1.81 \pm 0.1$ & $2.13 \pm 0.1^{*}$ \\
\hline VE/VCO ${ }_{2}$ Slope- all data & $31.1 \pm 0.9$ & $27.6 \pm 0.6^{*}$ & $32.0 \pm 0.4$ & $29.6 \pm 0.4^{*}$ \\
\hline$\Delta \mathrm{PeTCO}_{2}$ (rest-exercise) & $3.8 \pm 0.6$ & $5.9 \pm 0.4^{*}$ & $3.6 \pm 0.8$ & $6.3 \pm 0.9^{*}$ \\
\hline
\end{tabular}

Data are reported as means \pm SE. Pre: pre-intervention; Post: post-intervention; CET: continuous exercise training; IET: interval exercise training; BMI: body mass index; RER: respiratory exchange ratio; HR: heart rate; $\mathrm{VO}_{2} \mathrm{VAT}$ : oxygen consumption at ventilatory anaerobic threshold; OUES: oxygen uptake efficiency slope; $\triangle \mathrm{PeTCO}$ : difference in end-tidal carbon dioxide pressure from rest to its highest value during exercise. ${ }^{*} \mathrm{P}<0.05$, compared to Pre (two-way ANOVA).

In addition to ventilatory efficiency, CAD patients had lower values for $\mathrm{VE} / \mathrm{VCO}_{2}$ Slope after either CET or IET than at baseline. In addition, after the interventions both groups demonstrated an increase in $\triangle \mathrm{PeTCO}_{2}$ at rest (Table 2). The increase in ventilatory efficiency after the interventions was similar between groups (Table 2).

The results of the correlation analysis between OUES and aerobic fitness after either CET or IET are shown in Figure 1. Significant associations were observed in both groups: 1) CET (OUES and $\mathrm{VO}_{2} \mathrm{VAT} r=0.57, \mathrm{P}=0.001$; OUES and Peak $V_{2} r=0.57, P=0.001$ ); 2) IET (OUES and $\mathrm{VO}_{2}$ VAT $r=0.80, \mathrm{P}=0.001$; OUES and $P$ eak $\mathrm{VO}_{2}$ $r=0.67, P=0.001)$. However, the OUES was not significantly correlated with ventilatory efficiency in either of the groups: CET (OUES and VE $/ \mathrm{VCO}_{2}$ Slope $r=-0.16, \mathrm{P}=0.33$ ); IET (OUES and VE $/ \mathrm{NCO}_{2}$ Slope $\mathrm{r}=-0.19, \mathrm{P}=0.29$ ).

After the interventions, both groups showed a reduction in ventilatory equivalent ratios for oxygen at matched work rates throughout the graded exercise test (Figure 2).

\section{Discussion}

The main findings of this study were: 1) CET and IET increased OUES and aerobic fitness in CAD patients;
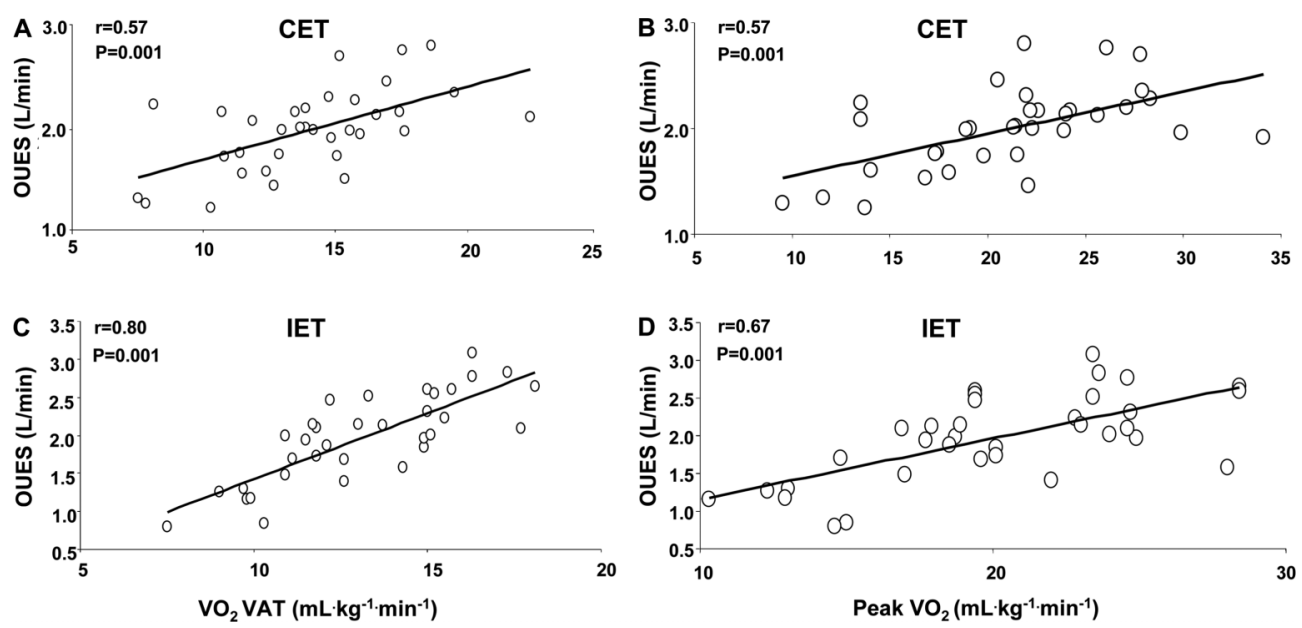

Figure 1. Relationship between oxygen uptake efficiency slope (OUES) and aerobic fitness in patients with coronary artery disease subjected to continuous exercise training (CET; $A$ and $B$ ) and interval exercise training (IET; $C$ and $D$ ). $\mathrm{VO}_{2}$ VAT: oxygen consumption at ventilatory anaerobic threshold; peak $\mathrm{VO}_{2}$ : peak oxygen consumption. 


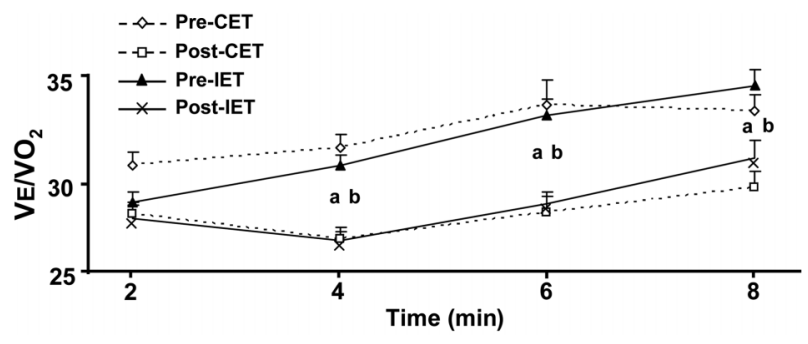

Figure 2. Ventilatory equivalent ratios for oxygen $\left(\mathrm{VE} / \mathrm{VO}_{2}\right)$ at baseline (Pre), and after 3 months (Post) of continuous exercise training (CET) and interval exercise training (IET), in patients with coronary artery disease. ${ }^{\mathrm{a}} \mathrm{P}<0.05$, compared to pre-CET; ${ }^{\mathrm{b}} \mathrm{P}<0.05$, compared to pre-IET (two-way ANOVA).

2) CET and IET resulted in the same degree of increase in OUES; 3) significant associations were observed between OUES and aerobic capacity for CET and IET.

This study evaluated the effects of interval and continuous exercise on OUES in patients with CAD. Determining the mechanisms involved in increasing OUES after exercise training are beyond the scope of the present study; however, we suggest that factors related to peripheral metabolic adaptations, such as increased aerobic metabolism, may be involved. After 12 weeks of either CET or IET, CAD patients demonstrated similar increases in $\mathrm{VO}_{2}$ at VAT. In addition, our results showed significant post-intervention associations between OUES and $\mathrm{VO}_{2}$ at VAT.

Baba et al. (2) proposed that the physiological mechanisms reflected in the OUES are the development of metabolic acidosis, which is controlled by distribution of blood to skeletal muscles, and also the physiologic dead space, which is affected by perfusion of the lungs. Thus, an increase in OUES after aerobic exercise training indicates that a given oxygen uptake has been achieved with lower ventilatory demand at submaximal workloads (16). Our results showed that the ratio of $\mathrm{VE}$ to $\mathrm{VO}_{2}$ (reflecting the ventilatory requirement for a given amount of work) was significantly reduced at matched work rates after CET and IET. Notably, an increase in capillary density, muscle blood flow, and mitochondrial density after aerobic exercise training leads to a delay in the onset of metabolic acidosis, thereby decreasing the ventilatory response during exercise. These results are in accordance with previous investigations demonstrating the effects of exercise training on OUES in cardiac patients $(6,16)$. For instance, Van Laethem et al. (6) showed an increase in OUES after a 6-month exercisetraining program in patients with chronic heart failure. Furthermore, in the same study, the authors observed that the increase in OUES correlated with improvement in VAT.

In the present study, after a 3-month exercise intervention, both training methods were found to result in the same level of improvement in aerobic fitness. These findings agree with those of previous studies that have demonstrated similar effectiveness of CET and IET for increasing cardiorespiratory fitness in CAD patients $(17,18)$.
However, other studies $(11,12)$ have observed a greater increase in fitness in response to IET than to CET. Rognmo et al. (11) reported a greater increase in cardiorespiratory fitness among CAD patients who performed IET, alternating between 4 min at higher intensity $\left(80 \%-90 \%\right.$ peak $\left.\mathrm{VO}_{2}\right)$ and 3 min at moderate intensity $\left(50 \%-60 \%\right.$ peak $\left.\mathrm{VO}_{2}\right)$ throughout the exercise session. In the same investigation (11), the CAD patients who performed CET completed $41 \mathrm{~min}$ of moderate intensity $\left(50 \%-60 \%\right.$ peak $\left.\mathrm{VO}_{2}\right)$ exercise for a 10 -week period. In contrast, in the present study the CAD patients in the IET group performed seven 3-min sets of exercise at RCP (80\%-90\% peak $\left.\mathrm{VO}_{2}\right)$ and seven 3-min sets of exercise at moderate intensity corresponding to the VAT, whereas the CET group patients performed 50 min of exercise at VAT $\left(70 \%-80 \%\right.$ peak $\left.\mathrm{VO}_{2}\right)$, for 12 weeks. We suggest that the similar fitness improvements observed in both groups may be attributed to the greater relative intensity and higher volume of exercise performed by the CET group in our present study. Controversy remains regarding the mode and intensity of aerobic exercise that yields optimal cardiorespiratory effects in CAD patients.

In this investigation, the improvement in OUES after aerobic exercise training was positively correlated with peak $\mathrm{VO}_{2}$. Similarly, Defoor et al. (16) showed an improvement in OUES in 425 patients with CAD after an exercise-training program. In the same study, the authors observed similar increases in both peak $\mathrm{VO}_{2}$ and OUES. These findings indicate that the OUES may be a sensitive measure for quantifying exercise performance improvements in cardiac patients.

It is important to mention that a previous study (19) reported that the use of beta-blockers could mitigate improvements in aerobic capacity in healthy subjects. In the present study, there were more patients using betablockers in the CET group than in the IET group. However, our results suggest that beta-blockers had no effect on the increase in aerobic capacity after exercise training in CAD patients. In the same context, Vanhees et al. (20) observed similar increases in peak oxygen uptake in CAD patients with and without beta blockers (36 and $34.5 \%$, respectively).

CET and IET showed a similar post-intervention ventilatory efficiency response, as shown by a decrease in VE/ $\mathrm{VCO}_{2}$ Slope. Additionally, both the CET and IET groups demonstrated an increase in $\triangle \mathrm{PetCO}_{2}$ at rest after the exercise intervention. Based on these findings, our results suggest that the increase in ventilatory efficiency after aerobic exercise training could be related to a reduction in ventilation/ perfusion mismatch (21). Furthermore, it has been suggested that increases in ventilatory efficiency after aerobic exercise training may be related to attenuation of hypercapnic $\mathrm{CO}_{2}$ chemosensitivity (22). However, in spite of these previous findings, we failed to observe a correlation between $\mathrm{VE} / \mathrm{VCO}_{2}$ Slope and OUES after the exercise intervention in either group. In fact, the present results suggest that the improvement in OUES after the interventions may not be associated 
with the physiological mechanisms related to the decreased $\mathrm{VENCO}$ Slope in CAD patients.

A limitation of this study is the fact that there were more hypertensive patients in the CET group than in the IET group. In general, hypertensive patients exhibit a greater increase in blood pressure during exercise than nonhypertensive subjects of the same age $(23,24)$. The higher systemic peripheral resistance associated with hypertension

\section{References}

1. Balady GJ, Arena R, Sietsema K, Myers J, Coke L, Fletcher GF, et al. Clinician's Guide to cardiopulmonary exercise testing in adults: a scientific statement from the American Heart Association. Circulation 2010; 122: 191-225, doi: 10.1161/CIR. 0b013e3181e52e69.

2. Baba R, Nagashima $M$, Goto $M$, Nagano $Y$, Yokota $M$, Tauchi N, et al. Oxygen uptake efficiency slope: a new index of cardiorespiratory functional reserve derived from the relation between oxygen uptake and minute ventilation during incremental exercise. J Am Coll Cardiol 1996; 28: 1567-1572, doi: 10.1016/S0735-1097(96)00412-3.

3. Hollenberg M, Tager IB. Oxygen uptake efficiency slope: an index of exercise performance and cardiopulmonary reserve requiring only submaximal exercise. J Am Coll Cardiol 2000; 36: 194-201, doi: 10.1016/S0735-1097(00)00691-4.

4. Marinov B, Kostianev S. Exercise performance and oxygen uptake efficiency slope in obese children performing standardized exercise. Acta Physiol Pharmacol Bulg 2003; 27: 59-64.

5. Tsuyuki K, Kimura Y, Chiashi K, Matsushita C, Ninomiya K, Choh $\mathrm{K}$, et al. Oxygen uptake efficiency slope as monitoring tool for physical training in chronic hemodialysis patients. Ther Apher Dial 2003; 7: 461-467, doi: 10.1046/j.15260968.2003.00084.x.

6. Van Laethem C, Bartunek J, Goethals M, Nellens P, Andries $E$, Vanderheyden M. Oxygen uptake efficiency slope, a new submaximal parameter in evaluating exercise capacity in chronic heart failure patients. Am Heart $J$ 2005; 149: 175180, doi: 10.1016/j.ahj.2004.07.004.

7. Davies LC, Wensel R, Georgiadou P, Cicoira M, Coats AJ, Piepoli MF, et al. Enhanced prognostic value from cardiopulmonary exercise testing in chronic heart failure by nonlinear analysis: oxygen uptake efficiency slope. Eur Heart $J$ 2006; 27: 684-690, doi: 10.1093/eurheartj/ehi672.

8. Hagberg JM. Physiologic adaptations to prolonged highintensity exercise training in patients with coronary artery disease. Med Sci Sports Exerc 1991; 23: 661-667, doi: 10.1249/00005768-199106000-00004.

9. Kendziorra K, Walther C, Foerster M, Mobius-Winkler S, Conradi K, Schuler G, et al. Changes in myocardial perfusion due to physical exercise in patients with stable coronary artery disease. Eur J Nucl Med Mol Imaging 2005; 32: 813-819, doi: 10.1007/s00259-005-1768-1.

10. Pattyn N, Coeckelberghs E, Buys R, Cornelissen VA, Vanhees L. Aerobic interval training vs moderate continuous training in coronary artery disease patients: a systematic review and meta-analysis. Sports Med 2014; 44: 687-700, doi: 10.1007/s40279-014-0158-x. at all levels of exercise may result in increased left ventricular workload and consequently an inadequate cardiac response during exercise.

In conclusion, our findings demonstrate that both CET and IET resulted in similar improvements in OUES in patients with CAD. These data suggest that improvements in OUES in CAD patients after aerobic exercise training may be dependent on both peripheral and central mechanisms.

11. Rognmo O, Hetland E, Helgerud J, Hoff J, Slordahl SA. High intensity aerobic interval exercise is superior to moderate intensity exercise for increasing aerobic capacity in patients with coronary artery disease. Eur J Cardiovasc Prev Rehabil 2004; 11: 216-222, doi: 10.1097/01.hjr.0000131677.96762.0c.

12. Wisloff U, Stoylen A, Loennechen JP, Bruvold M, Rognmo $O$, Haram PM, et al. Superior cardiovascular effect of aerobic interval training versus moderate continuous training in heart failure patients: a randomized study. Circulation 2007; 115: 3086-3094, doi: 10.1161/CIRCULATIONAHA.106. 675041.

13. Wasserman K, Whipp BJ, Koyl SN, Beaver WL. Anaerobic threshold and respiratory gas exchange during exercise. J Appl Physiol 1973; 35: 236-243.

14. Prado D, Dias R, Matos LDN. Fundamentos em avaliação da capacidade funcional cardiorrespiratória. In: Grave J, Raso V (Editors), Pollock fisiologia clínica do exercício. Barueri: Manole; 2012. p 11-29.

15. Tabet JY, Beauvais F, Thabut G, Tartiere JM, Logeart D, Cohen-Solal A. A critical appraisal of the prognostic value of the VE/VCO2 slope in chronic heart failure. Eur J Cardiovasc Prev Rehabil 2003; 10: 267-272, doi: 10.1097/00149831200308000-00008.

16. Defoor J, Schepers D, Reybrouck T, Fagard R, Vanhees L. Oxygen uptake efficiency slope in coronary artery disease: clinical use and response to training. Int J Sports Med 2006; 27: 730-737, doi: 10.1055/s-2005-872910.

17. Moholdt TT, Amundsen BH, Rustad LA, Wahba A, Lovo KT, Gullikstad LR, et al. Aerobic interval training versus continuous moderate exercise after coronary artery bypass surgery: a randomized study of cardiovascular effects and quality of life. Am Heart J 2009; 158: 1031-1037, doi: 10.1016/j.ahj.2009. 10.003.

18. Warburton DE, McKenzie DC, Haykowsky MJ, Taylor A, Shoemaker P, Ignaszewski AP, et al. Effectiveness of highintensity interval training for the rehabilitation of patients with coronary artery disease. Am J Cardiol 2005; 95: 1080-1084, doi: 10.1016/j.amjcard.2004.12.063.

19. Wolfel EE, Hiatt WR, Brammell HL, Carry MR, Ringel SP, Travis $\mathrm{V}$, et al. Effects of selective and nonselective betaadrenergic blockade on mechanisms of exercise conditioning. Circulation 1986; 74: 664-674, doi: 10.1161/01.CIR.74.4.664.

20. Vanhees L, Fagard R, Amery A. Influence of beta adrenergic blockade on effects of physical training in patients with ischaemic heart disease. Br Heart $J$ 1982; 48: 33-38, doi: 10.1136/hrt.48.1.33

21. Satoh T, Okano $\mathrm{Y}$, Takaki H, Matsumoto T, Yasumura $\mathrm{Y}$, Aihara $\mathrm{N}$, et al. Excessive ventilation after acute myocardial 
infarction and its improvement in 4 months. Jpn Circ $J$ 2001; 65: 399-403, doi: 10.1253/jcj.65.399.

22. Tomita T, Takaki H, Hara Y, Sakamaki F, Satoh T, Takagi S, et al. Attenuation of hypercapnic carbon dioxide chemosensitivity after postinfarction exercise training: possible contribution to the improvement in exercise hyperventilation. Heart 2003; 89: 404-410, doi: 10.1136/heart.89.4.404.
23. Amery A, Julius S, Whitlock LS, Conway J. Influence of hypertension on the hemodynamic response to exercise. Circulation 1967; 36: 231-237, doi: 10.1161/01.CIR.36.2.231.

24. Fagard RH, Pardaens K, Staessen JA, Thijs L. Prognostic value of invasive hemodynamic measurements at rest and during exercise in hypertensive men. Hypertension 1996; 28: 31-36, doi: 10.1161/01.HYP.28.1.31. 